\title{
Children's Reactions to Pain: A Psychiatrist's Perspective
}

\author{
Elva Orlow Poznanski, M.D.*
}

AIN and the reactions to pain are expressed in a diversiry of ways at a children's hospital. Pain becomes so closely linked with anxiety and fear that it is directly obvious how anxiety potentiates pain and how pain of itself promotes anxiety. Some children who have had little experience with pain appear startled when pain occurs, like an animal blinded by the headlights of a car. Occasionally, a child will use pain as an attention getting device, moaning in agony as soon as his parent walks through the door. Chronic pain complicating a chronic illness can promote regression in a child's behavior beyond what may be expected from the chronic illness itself.

In a burned child, pain appears to be the central factor in influencing his behavior. ${ }^{1}$ Pain appears to carry the primary responsibility for the feelings of anger, guilt, anxiety, depression, and the tendency to regression. Nover's five-year-old boy, who lacked sensation below the waist from birth and incurred a severe leg burn, did not demonstrate the characteristic behavior just described. He ate well and slept well throughout his hospitalization - a notable exception to most children with burns.

* Associate Professor of Psychiatry, Children's Psychiatric Hospital, University of Michigan Medicat Center, Ann Arbor, Mich. 48104.

\section{Reactivity to Pain in Infants and Young Children}

When looking at pain developmentally, one immediately comes to some very basic questions: At what age do infants feel pain? When are children able to localize pain? The answers to these simple questions are not totally known.

Pain cannot be readily measured. Like anxiety, it has a subjective quality that eludes precise definition. With adults, pain is often gauged by both verbal and nonverbal communications from the patient. With infants and toddlers, the difficulty is increased because one must rely entirely on nonlanguage observations.

When thinking about responses to pain in infants, we must bear in mind two important components: 1) the perception of pain itself; 2) the memory of past pain-the psychic factor.

The Neonate. During circumcision, babies in the first few days of life will commonly react to the clamping of the foreskin. Their reaction is a total body movement. The cries and screams associated with the procedure are generally brief and will cease on distraction. Indeed, some surgeons will operate on neonates without anesthesia for procedures that may last long periods of time. ${ }^{2}$ This is of considerable concern to an- 


\section{CHILDREN'S REACTIONS TO PAIN}

esthesiologists and child psychiatrists alike, since it is difficult to know whether the lack of anesthesia in the neonatal period has longterm psychologic consequences.

Multiple explanations have been proposed to explain the neonate's apparent indifference to pain. McGraw, ${ }^{3}$ who investigated the reaction of infants to pinprick, found that newborns did not exhibit any overt response to pimprick but did react to deep pressure sensation. But by the end of the first week or ten days, most infants will respond to cutaneous stimulation by diffuse bodily movements and crying. This reaction is immediate and increases in intensity during the first month.

Later in Infancy. After the first month, one can observe a noticeable decrease in diffuse body movements with crving. This diminution in response is followed by a phase that occurs roughly between three to ten months, in which the infant begins to localize the place being stimulated-for example, he will react to a pinprick by withdrawing the touched extremity. Only gradually does the ability to localize become more and more specific. McGraw noted that the child is able to show some general localization before he is able to recognize or associate the pin with a disagreeable sensation. Many anesthesiologists do not use general anesthesia until about three months of age, though there are no firm data on which to base this practice.

Escalona, well known for her extensive studies of infant development, notes that "neonates and very young infants differ from older babies in that they are both more and less reactive to sensory stimulation. A quiescent but alert and awake neonate may startle or show random motion in response to almost any sudden stimulus, whether it be sound, touch, vibration, or a bright light. Certain very slight stimuli, such as a small sound or a gentle touch on any portion of her skin will at times lead to circumscribed responses. . . such responses to the onset of external stimulation do not occur with regularity."

Escalona describes the very young infant as responding most to touch and passive motion. The relative importance of this response slowly diminishes, so that at five months the infant will demonstrate more overt behavior to visual and auditory components of the environment. This change may reflect the coordination and integration of stimuli rather than the type of stimulation. A four-month-old infant will react to two or more types of stimulation, e.g., he hears his mother's voice and turns his face to bring her into visual focus. This reponse may be medi-

Pain cannot be readily measured. Like anxiety, it has a subjective quality that eludes precise definition.

ated by better muscle tone and coordination, but there is also the possibility that since the infant selectively responds to the mother's voice the rudiments of memory may be emerging.

Indizidual Differences. Another aspect of both theoretic and practical importance relates to individual variations in the responses to pain. That such differences exist is well known, but in the older child and adult it is difficult to sort out the amount of physical pain itself from the host of other factors that collectively form the "psychogenic" or "mental" component. Escalona noted that individual differences in the response to perception of stimuli were easiest to identify between 4 to 12 weeks of age. Infants of 24 weeks or older no longer had the primary characteristics of simple reactivity, so that the responses could be described only in terms of complex and patterned behavior.

The infant of four months does not react simply to stimulation but his reactions are modified patterns that have emerged both from his innate perceptual response and the care-taking environment. Already at four months of age, the issue becomes complicated!

The various patterns of responses to complex stimuli have been studied by Stella Chess $^{5}$ in infants and young children. Chess uses the term "temperament" to describe those patterns that imply a response that is both innate and conditioned by the environment. 


\section{POZNANSKI}

\section{Recurrent Pain Syndromes in Young Children}

Recurrent pain syndromes on a psychogenic basis do not seem to arise clinically under the age of four years. Over the past half-decade, no patients of this type have been referred to psychiatry from pediatrics at the University of Michigan. Nor have such instances been recorded in the pediatric literature.

Older Children Who Complain of Recurrent Pain without Any Evident Organic Causation. These children are taken care of much more frequently in pediatric than in psychiatric clinics. Most of the articles about them can be found in pediatric journals. It is not surprising, therefore, that the literature on pain syndromes in children concentrates more on the physical status and statistics, and there is a relative dearth of descriptive behavioral data.

Some general characteristics of these children have emerged. They range in age from 5 to 16 years. The mean age for boys is 10.4 years; for girls, 11.5 years. ${ }^{6}$ There is no obvious peaking with puberty or adolescence. Large groups of these children have been tested for their intelligence and they are neither brighter nor duller than their peers. Other factors such as family size and ordinal position have not been found to be significantly different from the general population. These children do tend to come from families in which the parents are prone to pain.

Oster ${ }^{7}$ suggests that it is a "pain proneness and not the more specific bodily localization which shows a familial tendency." Apley, ${ }^{8}$ however, in his studies on children with recurrent abdominal pain, records the incidence of similar pains occurring at some time in the members of the immediate family as being six times higher than in the control children. In his children, the same site of pain in other family members was frequent.

With older children, recurrent abdominal pain is the most common pain complaint. Headaches are the next most frequent. ${ }^{6}$ Apley $^{8}$ was the first to note that a child who expresses one type of recurrent pain, e.g., headaches, is more likely to develop another type of recurrent pain, e.g., stomach aches, than a child who has never complained of recurrent pain in any site. He groups abdominal pain, headache, and "growing pains" together as expressions of a reactive pattern that is usually associated with emotional disturbance.

\section{Abdominal Pain}

Abdominal pain is the most frequent somatic complaint of children, yet most of the recurrent abdominal pains do not have an organic basis. Anxiety in a child is expressed more often as "I have a stomachache," than "I feel nervous or edgy." The latter phrase tends to be an adult expression. An example of the prevalence of stomach pains in childhood is demonstrated by Apley, ${ }^{10}$ who found that recurrent abdominal pain was reported in one out of nine school children. ${ }^{10}$ In pediatric clinics, only about 5 per cent of children brought in for recurrent abdominal pain have an organic origin for their pain. ${ }^{8}$

The child with recurrent abdominal pain is on the average three years younger than the child with recurrent headaches. The maximum age for such abdominal pain is nine years. It is difficult to explain this mean age difference. It could relate to the younger child's perception of his stomach as being an early and important part of his body image. The physiologic maturation of the body is an equally viable explanation.

The nature of the emotional difficulties that are associated with nonorganic abdominal pain is of considerable interest to the psychiatrist and the pediatrician. A pley ${ }^{8}$ described the emotional disturbances in these children as covering a wide variety of problems including fears, sleep difficulties, appetite difficulties, and difficulties at school. His overall impression was that, while none of these particular items was nonspecific, several often occurred together in a child with recurrent abdominal pain.

During the past three years at the University of Michigan Medical Center, 14 inpatient children on pediatric service were referred for psychiatric consultation with at least one of the complaints being abdominal pain. This number represents 6 per cent of all formal 


\section{CHILDREN'S REACTIONS TO PAIN}

referrals from pediatrics to psychiatry. In 13 of these 14 children, no organic basis had been found to account for the pain. The one child who did have an organic cause was a six-yearold with a duodenal ulcer.

Of these 13 children, four children were instances of classical school phobia or the School Refusal Syndrome. One other, although not so labeled, descriptively sounded like a school phobic. School phobia pain characteristically arises or is worse on Monday through Friday mornings before the start of school, and may be accompanied by vomiting. These children had all missed many weeks of school, disproportionate to the intensity of the somatic symptoms. Each one had been briefly admitted to a pediatric hospital by some physician concerned with their abdominal pain.

In six of the other eight, the abdominal pain children appeared to be a somatic expression of anxiety. For example, one tenyear-old girl had complained of abdominal pain for the previous two to three weeks-a period during which her mother had been hospitalized with renal calculi and infections. Besides the obvious possibility of identification with her mother, she expressed numerous concerns about her mother's health and her own impending vaginoplasty, since she had been born with an absent vagina. From the psychiatrists' interviews, it became clear that her abdominal pains expressed the fear of surgery that she consciously denied.

An abdominal pain, in addition to expressing anxiety, may yield some secondary gain. For example, one 12-year-old boy had abdominal pain of six weeks' duration for which no organic cause could be found. He was an

\section{Recurrent pain syndromes on a psychogenic basis do not seem to arise clinically under the age of four years.}

"A" student and high achiever, a "champion swimmer" until he was unable to compete because of the pain. His father had built a "competitive" 60 foot pool at home for his son to practice in. The boy was concerned that he would not be able to compete in swimming at just the point in his life where he would have the advantage of being the oldest in his division. Obviously, the pressure or anxiety around competition was disturbing the boy. The secondary gain from the abdominal pain was that it released him from competition. Secondary gain, when present as in this youngster in the school phobics, makes the recognition of a significant psychogenic component to the pain considerably easier.

With two of this group of children the etiology of their pain could not be decided

\begin{abstract}
Abdominal pain is the most frequent somatic complaint of children, yet most of the recurrent abdominal pains do not have an organic basis.
\end{abstract}

within the context of a brief evaluation. Some pain syndromes are not easily diagnosed and require extensive evaluation and follow-up.

One wonders what happens to children who have recurrent abdominal pains distressing enough to bring them to the pediatrician's office. One follow-up study has been done. Apley interviewed 30 such adolescents and young adults some eight to 20 years after they were initially seen for abdominal pain. ${ }^{9}$ During the intervening years, they were virtually an untreated group from a psychiatric perspective - that is, they did not receive any form of psychotherapy or counseling. At follow-up, roughly one-third were well; onethird had lost their abdominal pain but had other bodily symptoms; and one-third still had their recurrent abdominal pains, nearly always with additional symptoms. A similar follow-up study of the same type of patient, reported after the shorter time interval of two years, showed one-third remained the same and two-thirds were much improved or had entirely lost the symptom. ${ }^{6}$ This latter study did not look into the possibility of a shift in the site of the recurrent pain.

It is difficult to evaluate and measure the emotional component of pain. A possible physiologic accompaniment has been demonstrated by Apley, ${ }^{10}$ who noted that normal pupillary reactions are modified in children 


\section{POZNANSK!}

who have recurrent abdominal pain without physical cause and also in children with behavior problems. Such children took longer to recover from stress induced by a cold pressor test.

\section{Headache}

Clinically, headache is one of the commoner causes of absence from school in older children. Surveys vary as to the prevalence but roughly 5 per cent of school children seem to complain of frequent severe recurrent headaches.

As stated earlier, headaches tend to occur three years later than stomachaches, reaching a maximum occurrence at 12 years of age. ${ }^{7}$ It is not clear why this pain pattern tends to occur at a later age.

One of the common myths ${ }^{10}$ is that headaches in school children are caused by errors of refraction. Hence, a school child with a headache is often sent first to an ophthamologist. Actually, large numbers of children who have errors of refraction do not have headaches; further, when a refractive error does exist, wearing of glasses may help the eyesight without aiding the headache.

The pediatric and psychiatric literature lack papers describing the psychologic aspects of children with recurrent headaches. During the same three year period spent in the study of children with recurrent abdominal pain, three children with headaches were psychiatrically evaluated as inpatients at Mott Children's Hospital of the University of Michigan Medical Center. Such a small number indicates that children with headaches of psychologic origin are mainly treated as outpatients.

With each of these three children, extensive investigation uncovered no organic cause for their headaches. From a psychiatric perspective, these youngsters had two characteristics in common: 1) they had missed a great deal of time from school (for six weeks to three months continuously) - presumably because of their headaches. The secondary gain of missing school cannot be readily dismissed, and in this respect, they resemble the school phobic children, particularly those who complained of abdominal pain. 2) In psychiatric interviews, they were guarded and evasive, giving less information about themselves than other youngsters of: their age. For example, eleven-year-old Kathy had had unexplained headaches for two months. Her mother had a long history of migraine headaches. Kathy was extremely difficult to interview-frequently saying, "I don't know," or "there is nothing wrong with me," with an ever present smile. While three cases is too small a number to draw very basic generalizations from, it is possible to say that the child with headaches on an emotional basis may not readily perceive his own difficulties to be in the psychologic area.

\section{Limb Pain}

The concept of "growing pains" is about 100 years old. Physical growth when characterized by the three criteria of height, weight, and weight/height ratio does not have any inHuence on the incidence of growing pains. Hence the term "growing pain" is a poor term because of the lack of correlation between growth and pain. ${ }^{11}$

The frequency of growing pains is open to question. Studies vary widely, ranging from 4 to 18 per cent depending on the definition that may or may not include severity and frequency as a factor. ${ }^{12}$ The methods utilized in such studies are open to criticism. For example, in some surveys each child was directly asked if he had pain in his legs - "growing pains." Now, if one asks a child whether he has "growing pains" he is likely to answer affirmatively-simply because all children are deeply interested in growing and the question implies that some aches and pains may be associated with that process. Nevertheless, whatever term is used, pediatricians encounter children with recurrent limb pains, most of whom are not inconvenienced by their pains. No child with this pain syndrome has been referred from pediatrics to psychiatry in the last three years at our institution.

In the pediatric literature, the indications are that growing pains are complained of more frequently in pain-prone children, i.e., children who more likely have headaches and abdominal pain. ${ }^{9}$ One wonders about the 
prevalence of limb pains in certain types of children, such as the hyperactive child or the clumsy child or the child with flat feet. There are simply no data to affirm or deny such relationships.

\section{Final Comments}

In brief, the reactions of children to pain are not simple. The patterns are related to both the increasing maturity of the infant and young child and his capacity to communicate. Complicating the expressions in children, as in adults, anxiety and other emotional factors are often clear components of pain syndromes and may augment or distort the response to pain. In addition, some children tend to respond to pain in a style that is characteristic of their family.

Psychotherapy would seem to be helpful for long-range relief of pain that presumably is psychogenic in origin, but there are no controlled studies to substantiate this recommendation:

\section{References}

1. Nover. R. A.: Pain and the burned child. $J$. Amer. Acad. Child Psychiatry 12: 3, 1973

2. Smith, R. M.: Anesthesia for Infants and Children, 3rd ed. St. Louis, C. V. Mosby Co., 1968.

3. McGraw, M. D.: The Neuromuscular Maturation of the Human Infant. New York. Columbia University Press. 1943

4. Escalona, S.: The Roots of Individuality, Chicago, Aldine Publisting Co., 1968.

5. Thomas, A.. Chess, S., and Birch, H. G.: Temperament and Behavior Disorders in Children. New York, New York University Press, 1968

6. Friedman, R.: Some characteristics of children with 'psychogenic' pain. Clin. Pediatr. 11: 331. 1972.

7. Oster, $\mathrm{J}$ : Recurrent abdominal pain. headache and limb pains in children and adolescents. Pedatrics 50: 3,1972

8. Apley, J: The child with recurrent abdominal pain. Pediatr. Clin. North Am. 14: 1, 1967

9. Apley, J, and Mackeith, R.: The Child and His Symptoms, Oxtord \& Edinburgh, Blackwell Scientific Publications. 1968.

10. Apley. J. Haslam, D. and Tullock, Ca: Pupillary reaction in children with recurrent abdominal pain. Arch. Dis. Child 46:337, 1971.

11. Oster, J., and Nielsen, A.: Growing pains-a clinical investigation of a school popratation. Acta Paediat. Scand 61: 329,1972 . 